\title{
THE
}

$5-1-1990$

\section{Chaos in Spin Clusters: Quantum Invariants and Level Statistics}

Niraj Srivastava

Charles Kaufman

University of Rhode Island, ckaufman@uri.edu

Gerhard Müller

University of Rhode Island, gmuller@uri.edu

Follow this and additional works at: https://digitalcommons.uri.edu/phys_facpubs

Terms of Use

All rights reserved under copyright.

\section{Citation/Publisher Attribution}

Niraj Srivastava, Charles Kaufman and Gerhard Müller. Chaos in spin clusters: quantum invariants and level statistics. J. Appl. Phys. 67 (1990), 5627-5629.

Available at: http://dx.doi.org/10.1063/1.345907

This Article is brought to you for free and open access by the Physics at DigitalCommons@URI. It has been accepted for inclusion in Physics Faculty Publications by an authorized administrator of DigitalCommons@URI. For more information, please contact digitalcommons-group@uri.edu. 


\title{
Chaos in spin clusters: Quantum invariants and level statistics
}

Niraj Srivastava, Charles Kaufman, and Gerhard Müller

Department of Physics, The University of Rhode Island, Kingston, Rhode Island 02881-0817

\begin{abstract}
The energy-level sequence, whose spacings distribution is the most frequently invoked indicator of quantum chaos, can be derived (for systems with two degrees of freedom) from a two-dimensional representation of quantum invariants by projection. In this representation, such properties of level sequences as effective randomness in integrable models and level repulsion in nonintegrable models can be more directly interpreted in terms of physical properties. In integrable models, anharmonicities convert quasiperiodic level sequences into effectively random sequences.
\end{abstract}

While the frequently used term quantum chaos has still not completely emerged from obscurity, many quantum nonintegrability effects have been identified in recent years and are currently being studied in great detail.' Among them stands out the striking correlation between the fluctuation properties of the energy spectrum of a quantum model system and the (non-) integrability of the corresponding classical model. ${ }^{1-3}$ Quantitative descriptions of these fluctuations, primarily in terms of level-spacings distributions, exist for the cases of complete integrability and complete global chaos.

In this paper we shall report some insights into the origin of different energy-level statistics which have resulted from our ongoing study of chaos in spin clusters. ${ }^{4-6}$ All results presented here are for a system of two localized quantum spins interacting via an anisotropic exchange coupling and subject to crystal-field torques. Our model Hamiltonian reads

$$
\widehat{H}=\hbar^{2} \sum_{\alpha=x y z}\left\{-J_{\alpha z} \hat{\sigma}_{1}^{\alpha} \hat{\sigma}_{2}^{\alpha}+\frac{1}{2} A_{\alpha}\left[\left(\hat{\sigma}_{1}^{\alpha}\right)^{2}+\left(\hat{\sigma}_{2}^{\alpha}\right)^{2}\right]\right\} .
$$

In the limit $\hbar \rightarrow 0, \sigma \rightarrow \infty, \hbar \sqrt{\sigma(\sigma+1)}=s, \hbar \hat{\sigma}_{1}^{\alpha}=S_{1}^{\alpha}, \hat{H}$ turns into the energy function $H\left(\mathbf{S}_{1}, \mathrm{~S}_{2}\right)$ of an autonomous classical Hamiltonian system of two degrees of freedom for two three-component vectors of length $s, \quad \mathbf{S}$ $=s\left(\sin \vartheta_{l} \cos \varphi_{l} \sin \vartheta_{l}, \sin \varphi_{l}, \cos \vartheta_{l}\right)$, specified by the equation of motion $d \mathbf{S}_{l} / d t=-\mathbf{S}_{l} \times \partial H / \partial \mathbf{S}_{l}$. Each classical spin $\mathbf{S}$, is expressible in terms of a pair of canonical coordinates $p_{l}=s \cos \vartheta_{l}, q_{l}=\varphi_{l}$. The classical two-spin system is completely integrable if there exists an independent integral of the motion in addition to $H$. In a previous study ${ }^{4}$ we have determined the integrability condition

$$
\begin{aligned}
\left(A_{x}\right. & \left.-A_{y}\right)\left(A_{y}-A_{z}\right)\left(A_{z}-A_{x}\right) \\
& +\sum_{\alpha \beta \gamma} \sum_{\operatorname{cycl}(x y z)} J_{\alpha}^{2}\left(A_{\beta}-A_{\gamma}\right)=0
\end{aligned}
$$

and have constructed explicitly the second independent integral of the motion $I\left(\mathbf{S}_{1}, \mathbf{S}_{2}\right)$ for the integrable cases. The goal here is to gain further insight into the connection between (a) the manifest dependence of the energy-level statistics on the integrability property of the underlying system and (b) the presence or absence of well-understood dynamical phenomena associated with Hamiltonian chaos. For integrable and near-integrable systems, it is useful and natural to look at the energy level sequence as the projection onto a line (the energy axis) of a two-dimensional (2D) representation of quantum invariants. In this representation, the origin of the physical effects which give rise to different level spacings distributions are more directly accessible to intuitive understanding and quantitative analysis.

Integrability of the classical two-spin cluster implies that the flow in four-dimensional (4D) phase space is confined to 2D tori. The phase space is densely foliated by such invariant tori. Each torus is specified by the values of two action variables $J_{1}, J_{2}$, and the two independent analytic invariants are functions of these two action variables: $H\left(\mathrm{~S}_{1}, \mathrm{~S}_{2}\right)=E\left(J_{1}, J_{2}\right), I\left(\mathrm{~S}_{1}, \mathrm{~S}_{2}\right)=I^{\prime}\left(J_{1}, J_{2}\right)$. The corresponding quantum energy spectrum $E_{\lambda}$ is then naturally catalogued as a two-parameter family in terms of quantum numbers $m_{1}, m_{2}$, each representing one of two action variables $J_{k}=m_{k} \hbar, m_{k}=-\sigma,-\sigma+1, \cdots, \sigma$ for $k=1,2$ in accordance with semiclassical quantization.

This implicit 2D order of the eigenvalue sequence $E_{2}$ in terms of the two quantized action variables can be displayed even if we do not know the function $E\left(J_{1}, J_{2}\right)$ explicitly: Consider the energy eigenvalues $E_{\lambda}$ and the eigenvalues $I_{\lambda}$ of the quantum invariant $\widehat{I}$, the quantum version of $I\left(\mathbf{S}_{1}, \mathbf{S}_{2}\right)$. In a diagram $I_{\lambda}$ vs $E_{\lambda}$, the $2 \mathrm{D}$ array of points then form a regular pattern, which, however, is likely to be visually distorted due to the generally complicated nonlinear dependence of $E$ and $I^{\prime}$ on $J_{1}, J_{2}{ }^{6}$ We expect that the points representing any particular symmetry class of eigenstates can be connected to a fully intact $2 \mathrm{D}$ web with four bonds per vertex. Locally, the web should resemble a square lattice with spacing of $O(\hbar)$. Figure 1 (a) shows such a web. There we have plotted the eigenvalues $I_{\lambda}^{(M)}$ of the second invariant $\hat{I}_{M}$ (to be specified) versus the energy eigenvalues $E_{\lambda}$ for the integrable case $J_{x}=1.2, J_{y}=0.8, \quad J_{z}=0, A_{x}=A_{y}$ $=A_{z}=0$ of the two-spin model (1). The vertices represent all eigenvalues of those states of $\hat{H}$ ( for $\sigma=35, s=1$ ) which transform according to the irreducible representations A1A and B1S of the symmetry group $D_{2} \otimes S_{2}$, where $S_{2}$ is the permutation group of the two spins and $D_{2}$ contains all twofold rotations about the coordinate axes. ${ }^{7}$ Each vertex in the web represents a quantized torus claiming an area $\Delta \Omega \approx \hbar^{2}$, the size of one mesh, in accordance with the uncertainty principle. Connecting the points by bonds to a complete web amounts to assigning a pair of quantum numbers to each 

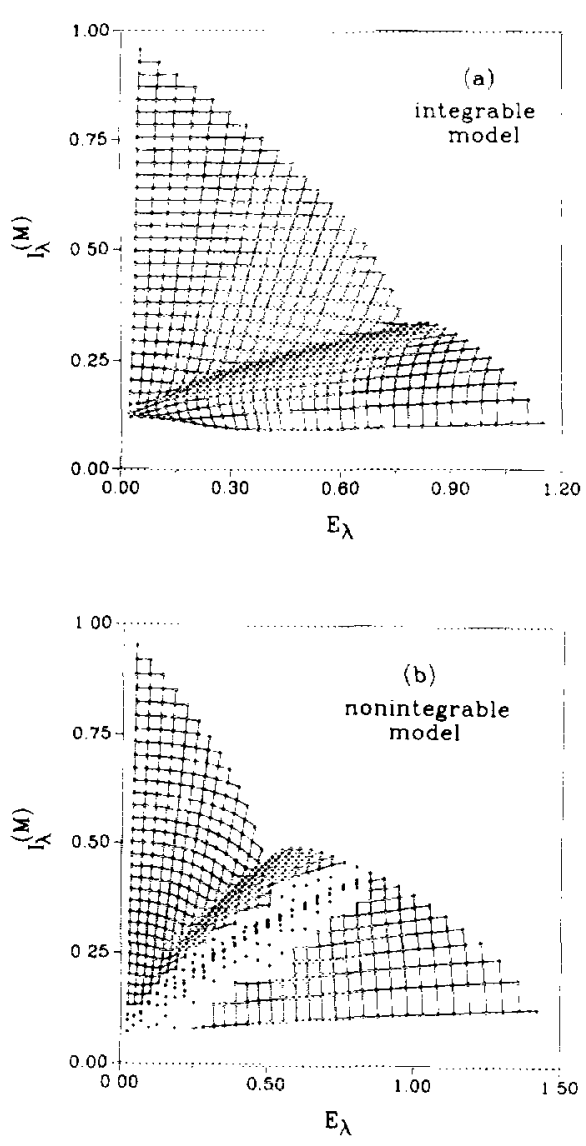

FIG. 1. Quantum invariant $I_{A}^{\text {v }}$ vs energy $E$, for all eigenstates (with $\left.E_{,}>0\right)$ of symmetry clasts A A And B1S of two special cases of the quantum two-spin model (1) for $\sigma=35, s=1:$ (a) integrable model $J_{1}=1.2$ $J_{v}=0.8 . J_{z}=0, A_{1}=A_{v}=A_{z}=0$; (b) nonintegrable model $J_{1}=J_{v}=1$, $J_{z}=0, A_{1}=-A_{v}=-0.5, A_{z}=0$.

eigenstate, i.e., chonsing a particular sot of action variables.

We could have constructed our second invariant $\hat{I}$ directly from the classical integral of the motion as derived in Ref. 4. Instead we pick up Peres' ${ }^{8}$ idea and construct $\hat{I}$ from some arbitrary dyramical variable vis time average ${ }^{6}$ : Take any dynamical variable $\widehat{A}$ which is independent of $\widehat{H}$ (e.g., choose $\hat{A}$ such that $[\widehat{A}, \hat{H}] \neq 0$ ), and consider the matrix elements of $\hat{A}(t)$ in the energy representation, $\left\langle\lambda|\widehat{A}(t)| \lambda^{\prime}\right\rangle$ $=A_{\lambda \lambda} \cdot \exp \left[\left(E_{\lambda} \cdots E_{\lambda} \cdot\right) t / \hbar\right]$. Performing the time average wipes out all off-diagonal elements and thus defines the quantum invariant $\hat{I}_{A}$, which is diagonal in the energy repre sentation as it should be: $\left\langle\lambda|\widehat{A}(t)| \lambda^{\prime}\right\rangle$ $=\langle\lambda|\hat{A}| \lambda\rangle \delta_{\lambda \lambda}$. $I_{\lambda}^{(A)} \delta_{\lambda \lambda}$. In the case where degenerate energy levels occur, the eigenvectors in the invariant subspaces must be chosen such that all off-diagonal elements $A_{\lambda \lambda}$ are zero. Throughout this study we use the quantum invariant

$$
I_{\lambda}^{(M)}=\sqrt{\left(\lambda\left|\hat{M}_{z}^{2}\right| \lambda\right\rangle}, \quad \hat{M}_{z}=\hbar\left(\hat{\sigma}_{1}^{z}+\hat{\sigma}_{2}^{z}\right) / 2,
$$

which is, for the integrable cases of $(1)$, a valid substitute for the invariant operators derived from the explicitly known classical analytic invariant $I\left(S_{1}, S_{2}\right)$. However, the quan-
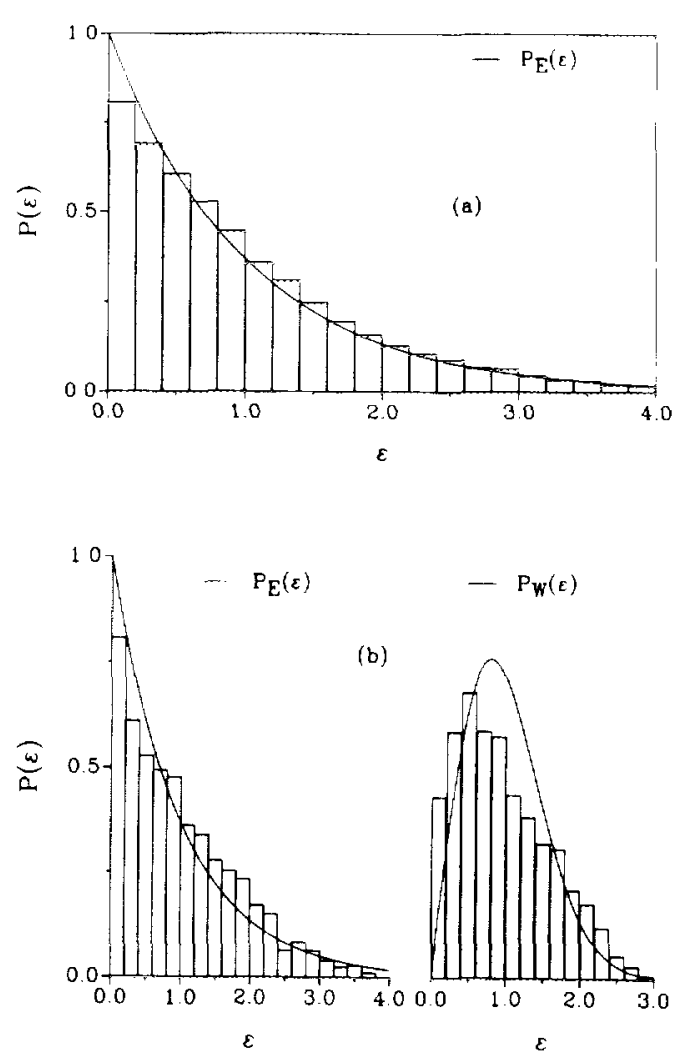

FIG. 2. Histogram plots of level spacings distributions (a) for the integrable model $J_{1}=1.2, J_{1}=0.8, J_{z}=0, A_{1}=A_{v}=A_{z}=0$ (8115 states were used for statistics) and (b) for the nonintegrable model $J_{1}=J_{v}=1$, $J_{:}=0, A_{1}=-A_{1}=-0.7, A_{z}=0$ (two windows of 6791 and 3724 states from the quantum web corresponding to a regular region and a chaotic region in phase space, respectively). The solid lines represent the exponential distribution $P_{1}(\epsilon)$ and the Wigner distribution $P_{11}(\epsilon)$ for comparison. The data are for systems with $\sigma=65$.

tum invariant ( 3 ) can also be constructed for nonintegrable cases of our two-spin model, i.e., for cases where the classical analytic invariant $I\left(\mathbf{S}_{1}, \mathbf{S}_{2}\right)$ does not exist. Not surprisingly, the properties of $I_{\lambda}^{(M)}$ depend sensitively on whether the classical integrability condition is satisfied or not. Figure 1 (b) shows a 2D representation of the quantum invariants $I_{\lambda}^{(M)}$ vs $E_{\lambda}$ for the nonintegrable model specified by parameters $J_{x}=J_{y}=1, J_{z}=0, A_{x}=-A_{y}=-0.5, A_{z}=0$. We observe two large regions in which the web of quantized tori is fully intact. In between, the web is clearly interrupted by a broad band of chaos. Classical Hamiltonian chaos, which is dense everywhere in phase space, does not manifest itself in quantum mechanics as long as the chaotic regions are small compared to the mesh size $\hbar^{2} \approx s^{2} / \sigma^{2}$ of the quantum web. This describes the situation in the two regular regions of Fig. 1 (b). If the chaotic region is sufficiently large, however, or the mesh size sufficiently small, then quantum nonintegrability effects are indeed quite dramatic in appearance. Within the chaotic region, the quantum states tend to cluster in short strips along one of the interrupted lines of constant action, leaving sizeable areas of the web depleted of states. 
Within any one of these small clusters, the quantum states are slightly displaced sideways, enough to account for the effect of level repulsion after projection onto the $E_{\lambda}$ axis.

The $2 \mathrm{D}$ representation of quantum invariants has the advantage of making it possible to sample parts of the total spectrum for energy-level statistics in a very controlled manner and of enabling us to identify the nature of the classical phase flow associated with those individual parts. We have selected one window of states from the quantum web of the integrable model $J_{x}=1.2, \quad J_{y}=0.8, \quad J_{z}=0$, $A_{x}=A_{y}=A_{z}=0$, and two windows of states from the $\left(I_{\lambda}^{(M)}, E_{\lambda}\right)$ representation of the nonintegrable model $J_{x}=J_{y}=1, J_{z}=0, A_{x}=-A_{y}=-0.7, A_{z}=0$, one each in a regular and a chaotic region. For states within each one of the three windows we have determined the level spacings distribution $P(\epsilon)$. That analysis was performed for systems with $\sigma=65$, yielding a total of 17161 states. The $E_{\lambda}$ sequences were unfolded to take into account the nonuniform classical energy density. The results, averaged over all eight classes of the symmetry group $D_{2} \otimes S_{2}$, are displayed in Fig. 2 in the form of histograms. For comparison we have included as solid lines the exponential distribution function $P_{E}(\epsilon)=\exp (-\epsilon)$, which is predicted to describe the data for an integrable model, and the Wigner distribution function $P_{w}(\epsilon)=(\pi \epsilon / 2) \exp \left(-\pi \epsilon^{2} / 4\right)$, which is expected to describe the data for a globally chaotic model. ${ }^{1,3}$ Our results for the three windows of states are in good qualitative agreement with the theoretical predictions. There is not a hint of level repulsion in the regular regions of the nonintegrable model, but in the chaotic window that phenomenon is almost as pronounced as one might expect for random matrices.

The $2 \mathrm{D}$ representation of quantum invariants also provides the basis for an intuitive understanding of the different level-spacings statistics: Level repulsion is a general phenomenon for states of a particular symmetry class, irrespective of whether the system is integrable or not. In integrable models and in regular regions of chaotic models, level repulsion is a $2 \mathrm{D}$ phenomenon, manifesting itself in the quantum web. Upon projection of the states onto the energy axis, the effect of $2 \mathrm{D}$ level repulsion is totally lost in the level-spacings distribution $P(\epsilon)$, if not necessarily so in more sensitive statistical tools. In chaotic regions of nonintegrable models, on the other hand, level repulsion is a $1 \mathrm{D}$ phenomenon to begin with, because of the presence of only one quantum number, and is therefore unaffected by the projection of the states onto the $E_{\lambda}$ axis.

We conclude this report with a remark on the important role of anharmonicities for the energy-level statistics. ${ }^{9}$ Consider the classical energy function $E\left(J_{1}, J_{2}\right)$ for an integrable case of our two-spin model (1). Pick any invariant torus specified by action variables $J_{1}^{0}, J_{2}^{0}$ and use it as a reference point for semiclassical quantization. In the neighborhood of the point $\left(J_{1}^{0}, J_{2}^{0}\right)$ on the action plane, the function $E\left(J_{1}, J_{2}\right)$ can then, upon semiclassical quantization, be expanded in powers of $\hbar$ as follows ${ }^{8}$ :

$$
E_{m_{1} m_{2}}=E\left(J_{1}^{0}, J_{2}^{0}\right)+\hbar \sum_{k} m_{h} v_{h}
$$

$$
+\frac{1}{2} \hbar^{2} \sum_{k k} m_{k} m_{k} \cdot w_{k k}+\cdots,
$$

where $v_{k}=\partial E / \partial J_{k}, \quad w_{k^{\prime}}=\partial^{2} E / \partial J_{k} \partial J_{k^{\prime}}, k, k^{\prime}=1,2$, etc., and the $m_{k}$ are unconstrained integers. The quantum states on the $\left(E_{\lambda}, I_{\lambda}\right)$ plane thus obtained from (4) and a corresponding expansion for the second analytic invariant $I\left(J_{1}, J_{2}\right)$ then form an infinite array which approximates, in the vicinity of $\left(J_{1}^{0}, J_{2}^{0}\right)$, the exact quantum web discussed previously. For an analysis of the associated level statistics, set $v \equiv v_{2} / v_{1}$ and consider the distribution of the rescaled energy levels, $\bar{E}_{m_{1} m_{2}} \equiv E_{m_{1} m_{2}} / v_{1}$, on the interval $0<\bar{E}_{m_{1} m_{3}}<1$. To $O(\hbar)$ that distribution is equivalent to the distribution of the fractional part of $n v, n=1, \cdots, N$, where $N$ is proportional to $M$ for $\left|m_{1}\right| \leqslant M,\left|m_{2}\right| \leqslant M$. If $v=p / q$ is a rational number, only a single spacing (of size $\epsilon=1 / q$ ) occurs provided $N \geqslant q$. If $v$ is an irrational number, all levels are nondegenerate and spread uniformly over the interval. The counterintuitive result, however, is that the number of different level spacings (on the unit interval wrapped around to a ring) never exceeds 3 , no matter how large $N$ is. ${ }^{10}$ This result holds for all irrationals. While the resulting spacings distribution $P_{N}(\epsilon)$ thus simply consists of three $\delta$ functions, there is, in fact, a considerable amount of complexity in the spacings sequence, which strongly depends on the choice of the irrational number $v$. For example, if $v=(\sqrt{5}+1) / 2$, the golden mean, and $N$ is a Fibonacci number, then only two distinct spacings occur and form a perfect Fibonacci sequence. As it turns out, any such peculiar and highly singular distribution of level spacings is extremely unstable against slight perturbations. If one introduces a weak modulation in $v$ such as is expected from terms $O\left(\hbar^{2}\right)$ in expansion (4) and attributable to anharmonicities in the classical time evolution, $P_{N}(\epsilon)$ collapses immediately into a continuous distribution. For almost all types of perturbations, this is the exponential distribution $P_{E}(\epsilon)$. In other words, the slightest anharmonicities in the (integrable) classical Hamiltonian, transform a quasiperiodic sequence of energy levels into an effectively random sequence.

This work was supported in part by the U.S. National Science Foundation Grant No. DMR-86-03036 and by Sigma Xi, the Scientific Research Society. The numerical calculations were performed on the CRAY-2 of the National Center for Supercomputing Applications, University of Illinois at Urbana-Champaign.

'B. Eckhardt, Phys. Rep. 163, 205 (1988), and references therein

'O. Bohigas, M. J. Giannoni, and C. Schmit. Phys. Rev. Let1. 52. 1 ( 1984 ).

'J. V. José, in Directions in Chaos, edited by Hao Bai-Lin (World Scientific, Singapore, 1988), Vol. II.

${ }^{4}$ E. Magyari, H. Thomas, R. Weber, C. Kaufman, and G. Müller, Z. Phys. B 65, 363 (1987).

'N. Srivastava, C. Kaufman, G. Müller, R. Weber, and H. Thomas, Z. Phys. B 70, 251 (1988).

'N. Srivastava and G. Muller, Quantum Images of Hamiltonian Chaos (to be published).

'P. W. Atkins, M. S. Child, and C. S. G. Phillips, Tables for Group Theory (Oxford University Press, London, 1970)

${ }^{*}$ A. Peres, Phys. Rev. Lett. 53, 1711 ( 1984).

${ }^{3}$ M. V. Berry and M. Tabor, Proc. R. Soc. London Ser. A 356, 375 (1977) have discussed this aspect from a different vantage point.

"This curiosity had also been noticed by A. Pandey, O. Bohigas, and M. J. Giannoni, J. Phys. A 22, 4083 (1989). 